\title{
Research on Hybrid Picking Strategy in an Automated Order Picking System
}

\author{
Debao Liu ${ }^{1}$, Shandong Mou ${ }^{1}$, Yaohua $\mathrm{Wu}^{1}$, Guofeng Shan ${ }^{2}$ \\ ${ }^{1}$ School of Control Science and Engineering, Shandong University \\ ${ }^{2}$ YANTAI LongYuan Power Technology Co.,LTD. \\ debao.liu@163.com,msd12270711@gmail.com,mike.wu@263.com, \\ shanguofeng@lypower.com.cn
}

\begin{abstract}
In order to improve the picking efficiency of the automated order picking system based on catapult devices and reduce picking machine's waiting time caused by sequential order picking strategy, a hybrid picking strategy and its algorithm are proposed in this article. This algorithm conforms to the basic principle that every item does not overlap mutually on the conveyor system. The main step is to preprocess all picking orders. Firstly, non-interference items are selected to fulfill order picking in parallel. In order to reduce the gap caused by the first step on the conveyor system, the remaining items which can be picked precisely in the gap are selected then. In the end, the rest of items are picked based on sequential picking strategy. This hybrid strategy and its algorithm can ensure not only that all picked items are well-organized on the conveyor system, but also that the gap between items is reduced greatly. In the end of this article, the practical business data is applied for simulation analysis for the hybrid picking strategy and the sequential picking strategy. The results prove the hybrid picking strategy can greatly improve the picking efficiency and workload equilibrium of the automated order picking system.
\end{abstract}

Keywords: Automated order picking system, Order picking strategy, Hybrid picking strategy, Order Picking efficiency

\section{Introduction}

Nowadays the automated order picking system based on catapult devices is widely used in large retail distribution centers for medicine and tobacco industries, which is suitable for customer orders characterized by multiple species, small batch and regular shape, on account of its higher order picking efficiency, fewer error rates, and lower labor cost [1-2].

Automated order picking systems can be divided into several kinds based on the compound mode of sorting machines and conveying systems. As to sorting machine, one or several kinds are offered to be configured while unilateral or bilateral layout could be chosen [3-5]. Meanwhile, we can configure single or multiply conveying systems for parallel order picking. Generally, the system which is unilateral layout and single item sorting is called basic automated order picking system (BAOPS). In this paper, we choose BAOPS as the research target [6].

Various picking strategies could be chosen according to product quality, system structure and picking demand in automated order picking systems. Mainly, there are two kinds of picking strategies in continuous conveying systems, such as belt conveyor system etc. The first picking strategy is synchronous picking strategy or parallel picking strategy (PPS) [7-8], which means that various kinds of items in one single customer order are picked to the conveying system at the same time and the next order will not be dispatched to BAOPS until the previous one is fulfilled. It is suitable for small items with 
different shape that will not block up the conveying system, such as tablets. This picking strategy leads to higher efficiency, but items could not be packaged directly and are not easy to count. The second one is sequential picking strategy (SPS) [9], which means that picking sequence of customer orders must be in accordance with the display sequence of picking machine and the next customer order will not be dispatched to BAOPS until the previous one is fulfilled. It is suitable for picking items that need to be in regular sequence for packing after sorting process, such as cigarettes [10-11]. This picking strategy is not high-efficient but easy to pack and count. In the context of the discrete conveying system, it is suitable for items with small demand in multiple parallel order picking.

R. D. Meller and J. A. Pazour's research on automated order picking system was to regard the sorting machine as a mechanical picker [12]. They optimize item allocation problem by means of heuristic algorithm to decide optimal quantity of items. Sequential picking strategy has been widely used in automated order picking systems. Yingying $\mathrm{Wu}$ and Yaohua Wu optimized the total gap of items based on sequential picking strategy and dynamic virtual window algorithm (D-VWA) instead of static virtual window algorithm (S-VWA), which has been applied to double conveying systems [13]. Moreover, they built the buffer channel to reduce total gap of items in every picking machine, and then presented a mathematical model for automated order picking system based on compressive dynamic virtual window algorithm (CD-VWA).

Currently, it is common to optimize items sorting partition or order picking batch for picking efficiency improvement by means of transforming system hardware configuration, meanwhile less literature focused on picking strategy and its algorithm can be found. In this paper, we choose BAOPS as research target and do research on hybrid picking strategy (HPS). This hybrid picking strategy consists of PPS and SPS. The new hybrid picking strategy brings evident increase of picking efficiency for BAOPS.

\section{Automated Order Picking Systems}

There are several kinds of automated order picking systems, which consists of picking unit, conveying unit, packing unit and control unit. The system structure for BAOPS is showed in Figure 1.

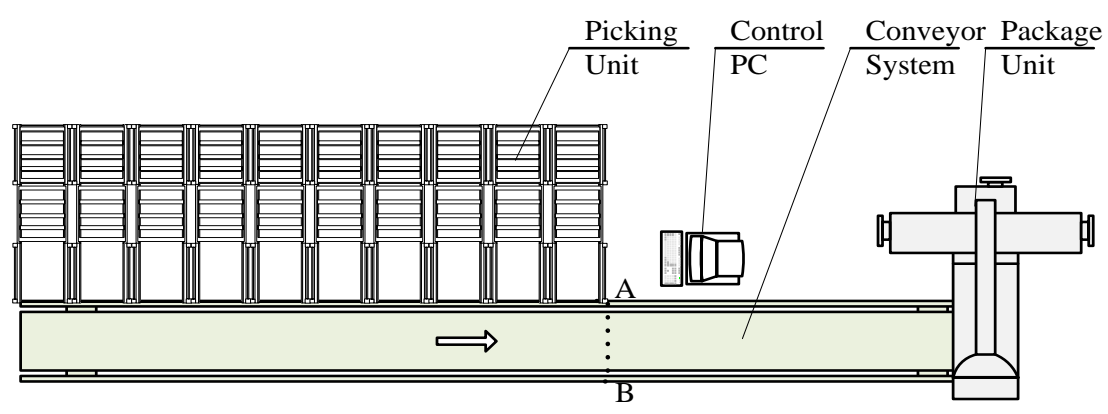

Figure 1. System Structure of BAOPS

While BAOPS is working, picking unit will pick required items to conveying unit. Then conveyor system carries items to the packing unit where all items are packaged into standard unit. The next customer order cannot be performed until the previous one is already finished.

In order to facilitate further research, let us make the following assumptions for BAOPS,

(1) Each picking unit is only responsible for one kind of items. Different picking unit represents different kinds of items. All picking items have the similar shape and size. 
(2) Picking unit could only regularly pick an item to conveying unit in one time. All items are put on the conveyor system in right assignment.

(3) There exists no stock out for each kind of items during picking process.

(4) All items in the similar category and the same order must be arranged in one area on the conveyor system. Besides, the speed of conveyor system and the speed of picking unit are reasonably matching, which guarantees that all items will not overlap.

(5) The speed of packing unit and the speed of picking unit must be also matching.

Order picking time is the main performance indicator to measure the efficiency of automated order picking system. Taking no account of the difference in the aspect of hardware capacity, order picking strategy and the coming algorithm design has become the key factor for system performance. In this paper, a new hybrid order picking strategy and its algorithm are proposed to improve the picking efficiency in BAOPS.

\section{Hybrid Order Picking Strategy}

While BAOPS conforms to SPS, the latter item is always waiting to be picked until previous items have been already picked to the conveyor system, which leads to much more waiting time compared with PPS. Accumulated waiting time has a vast influence on picking efficiency, especially taking into account those items which are in small demand.

Compared with SPS, PPS probably will lead to items overlap on the conveyor system. Thus, we present HPS for BAOPS. As is shown in Figure 2, when one customer order arrives at BAOPS, it will be pre-processed by this algorithm based on HPS. First, this algorithm will confirm whether the customer order is suitable for PPS or not. If the customer order can be fulfilled partially or completely in parallel, BAOPS will pick this part of items of the customer order in priority. Then, this algorithm will confirm whether the gaps of items on the conveyor system which is caused by the previous parallel order picking are available for the rest of items preciously. If there are available gaps, BAOPS will continue to pick this part of items synchronously. At last, the rest part of the customer order will be performed based on SPS. All required items on the conveyor system will be carried out of picking area and be packed in the package area. The latter customer order will not come into BAOPS until the previous round is fulfilled. Therefore, every customer order can be divided into three batches. The first order picking batch is called Parallel Picking Part (PPP), the second batch is named by Gap Picking Part (GPP), and the last one is defined as Sequential Picking Part (SPP).

We define $\mathrm{s}(1 \leq s \leq N)$ as non-zero number of items in order $i$. All corresponding picking machines in BAOPS are located from left to right according to the ascending sequence of the total number of required items for each picking machines. The non-zero items set is denoted by $U$. The detailed steps are explained as follows:

Step 1: Determine decision criteria for PPP and the first picking batch set.

Let us indicate two adjacent non-zero items by $N z_{i} \in U 、 N z_{i-1} \in U \quad\left(N z_{i}>N z_{i-1}\right)$ and assume that $N z_{i}$ belongs to PPP. Then, the decision criteria whether $N z_{i-1}$ can join PPP or not is:

$$
\lambda_{N z_{z_{i-1}}-N z_{i}}=\frac{\left(N z_{i}-N z_{i-1}-1\right) \times l}{v}-\left(a_{i N \Sigma_{i}}-1\right) \times t^{\prime}
$$

where $a_{i N_{i}}$ is the demand quantity of item $N z_{i}$ in the order $i, t^{\prime}$ is the time period to pick one item, $l$ represents the distance between centers of two adjacent picking machines and $v$ represents the speed of the conveyor system.

If $\lambda_{N_{z_{i-1}-} N_{z_{i}}} \geq 0$, the non-zero item can be selected into the first picking batch. If $\lambda_{N_{z_{i-1}-} N_{z_{i}}}<0$, the non-zero item cannot be selected into the first picking batch. 
Let us assume that the rightmost non-zero item in BAOPS is always in the first picking batch and then is chosen as the benchmark to classify whether its left-side non-zero item can be picked simultaneously based on formula (1). If they cannot be picked simultaneously, this algorithm will move to the next left-side non-zero item. If they can be picked simultaneously, this algorithm will set this item as the new benchmark to evaluate the next non-zero item based on formula (1). Repeat this process until all non-zero items have been evaluated and the first picking batch $U_{1}\left(U_{1} \subset U\right)$ consist of these items that can be picked simultaneously.

Step 2: Calculate gaps of items of GPP on the conveyor system after finishing the first picking batch.

Let us define two adjacent items of PPP by $f_{j} \in U_{1} 、 f_{j-1} \in U_{1}\left(f_{j}>f_{j-1}\right)$. The gap between the last stock keeping unit (SKU) of item $f_{i}$ and the first SKU of item $f_{i-1}$ on conveying system is:

$$
\delta_{f_{j-1}-f_{j}}=\left(f_{j}-f_{j-1}-1\right) \times l-\left(a_{i f_{j}}-1\right) \times t^{\prime} \times v
$$

Step 3: Determine items of GPP which can be picked to gaps of PPP on the conveyor system.

As to unsorted items which are not in the first picking batch, we can make use of gaps on the conveyor system to pick some items precisely under certain conditions.

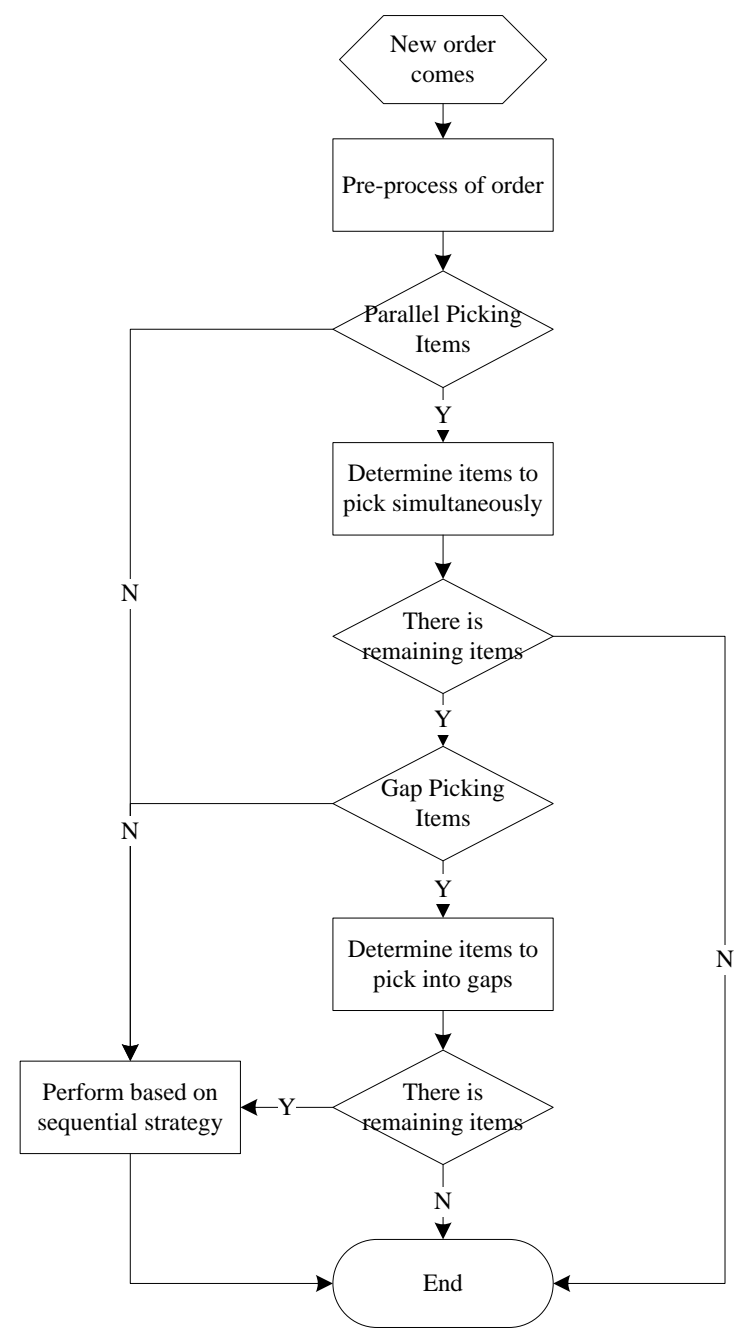

Figure 2. Flow Chart for the Hybrid Picking Strategy 
Let us define unsorted items by $\mathrm{g}(\mathrm{g} \in U)$. The certain condition whether item $g$ can be picked to gaps on the conveyor system which is formed by items $f_{j}$ and $f_{j-1}$ of PPP is

$$
\xi=\delta_{f_{j-1}-f_{j}}-\left[a_{i g} \times t^{\prime} \times v-\left(v \times t^{\prime}-l\right)\right]-\theta\left(f_{j}, g\right) \times\left(f_{j}-g-1\right) \times l
$$

where

$$
\theta\left(f_{j}, g\right)= \begin{cases}1, & f_{j}>g \\ 0, & f_{j}<g\end{cases}
$$

If $\xi \geq 0$, item $g$ can be picked to gaps on the conveyor system formed by items $f_{j}, f_{j-1}$. Furthermore, let us assume the start time for the first SKU of item $g$ is equal to the start time for the last SKU of item $f_{j}$ when $f_{j}>g$ and $g$ belongs to GPP. Otherwise, let us assume the start time for the first SKU of item $g$ is equal to the certain time that is the difference between the start time for the last SKU of item $f_{j}$ and the movement time on the conveyor system, $\left(v \times t^{\prime}-l\right)$, when $f_{j}<g$ and $g$ belongs to GPP.

Repeat the formula (3) one by one for all unsorted items that do not belong to PPP and then determine items of the second picking batch. Let $\mathrm{U}_{2}\left(U_{2} \subset U\right)$ denote GPP.

Step 4: Determine picking sequence of items of the third picking batch.

As to the remaining items, they will be picked in progressive sequence which is from left to right along with the conveyor system and the priority level is lower than PPP and GPP.

Let us define the far end of items of the first picking batch as $f_{1}\left(f_{1} \in U_{1}\right)$. Then the finish time of item $f_{1}$ picking is:

$$
t_{i_{-} f_{1}}^{e}=a_{i f_{1}} \times t^{\prime}
$$

Define the far end of unsorted item as $\operatorname{Tr}_{1}\left(T r_{1} \in U\right)$. Then the start time of $T r_{1}$ is:

$$
t_{i_{-} T_{\mathrm{1}}}^{s}=\max \left(\left(t_{i_{-} f_{\mathrm{1}}}^{e}-\frac{\left(f_{1}-T r_{1}\right) \times l}{v}\right), 0\right)
$$

And the finish time of $T r_{1}$ is:

$$
t_{i_{-} T_{r_{1}}}^{e}=t_{i_{-} T_{1_{1}}}^{s}+a_{i T_{1_{1}}} \times t^{\prime}
$$

Define the unsorted item on the right side of $\operatorname{Tr}_{1}$ as $\operatorname{Tr}_{2}$. According to formula (7), the start time of $T r_{2}$ is

$$
t_{i_{-} T_{r_{2}}}^{s}=t_{i_{-} T_{1_{1}}}^{e}+\frac{\left(T r_{2}-T r_{1}\right) \times l}{v}
$$

And the finish time of $T r_{2}$ is:

$$
t_{i_{-} T_{r_{2}}}^{e}=t_{i_{-} T_{r_{2}}}^{s}+a_{i T_{r_{2}}} \times t^{\prime}
$$

Define the total numbers of items in the third picking batch as $P$. According to formula (11) and (12), the start time of the last unsorted item which means the nearest item along with the conveyor system is: 


$$
t_{i_{-} T r_{P}}^{s}=t_{i_{-} T_{1}}^{s}+\sum_{p=1}^{P-1} a_{i T r_{p}} \times t^{\prime}+\frac{\left(T r_{P}-T r_{1}\right) \times l}{v}
$$

And the finish time of $\operatorname{Tr}_{P}$ is:

$$
t_{i_{-} T_{r_{P}}}^{e}=t_{i_{-} T r_{P}}^{s}+a_{i T_{P}} \times t^{\prime}
$$

Step 5: Calculate the total picking time for one single customer order.

Based on formula (5) (6) (10) (11), we can derive the total picking time for order $i$ :

$$
t_{i}=\max \left(\left(t_{i_{-} f_{1}}^{e}-\frac{\left(f_{1}-T_{1}\right) \times l}{v}\right), 0\right)+\sum_{p=1}^{P} a_{i T r_{p}} \times t^{\prime}+\frac{\left(T r_{P}-T_{1}\right) \times l}{v}+\frac{\left(N-T r_{P}\right) \times l}{v}
$$

\section{Simulation Analysis}

In order to verify HPS in the aspect of enhancing the picking efficiency in BAOPS, a complete customer orders case of a full working day in a tobacco logistics distribution center is chosen for simulation analysis. This case consists of 825 orders, 103 items and 42711 pieces of cigarette.

There exist 103 working channels in BAOPS. The speed of the conveyor system is $1 \mathrm{~m} / \mathrm{s}$. The center distance between adjacent working channels is $0.2 \mathrm{~m}$. And it spends $0.3 \mathrm{~s}$ to finish picking an item to the conveyor system. All items are arranged with working channels in ascending order from left to right based on ordering number.

We use MATLAB7.4 to analyze the effectiveness for SPS and HPS. The simulation results are shown in Table 1 . When the hybrid picking strategy is applied to BAOPS, the total order picking time has decreased by $25 \%$ and average picking efficiency has increased by $33.5 \%$, compared with the sequential picking strategy.

Figure 3 shows the comparison of accumulated picking time between SPS and HPS. With the increase of numbers of customer orders, it can save more working time based on the hybrid picking strategy.

Figure 4 illustrates the difference of instantaneous picking time between SPS and HPS. As is illustrated in Figure 4, all instantaneous values are greater than zero, which means picking time will be shorten under the condition of the hybrid picking strategy. The smallest reduction value is 0.8 second and the maximum reduction value is 20.6 seconds.

Table 1 Comparison Results of Two Methods Of Simulation

\begin{tabular}{ccc}
\hline & Sequential picking strategy & hybrid picking strategy \\
\hline Total picking time & $17674.1 \mathrm{~s}$ & $13235.5 \mathrm{~s}$ \\
Average picking efficiency & 8699.7 pieces/hour & 11617.2 pieces/hour \\
\hline
\end{tabular}




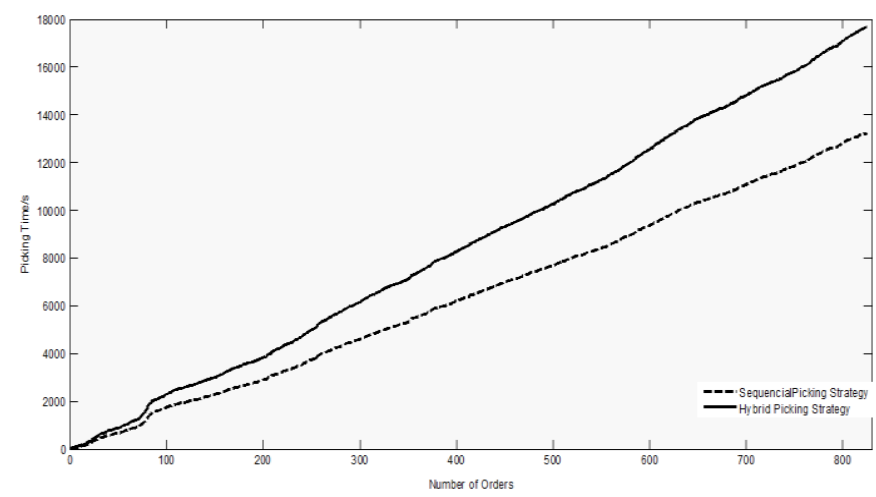

Figure 3. Accumulated Picking Time between SPS and HPS

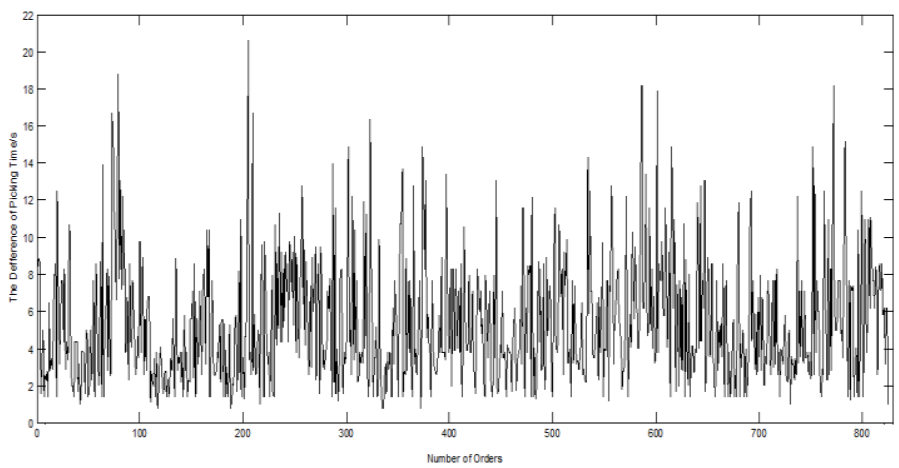

Figure 4. The Difference Of Instantaneous Picking Time Between SPS And HPS

Figure 5 shows the comparison of instantaneous picking efficiency and the average picking efficiency based on the sequential picking strategy and the hybrid picking strategy. It is evident that instantaneous picking efficiency and mean picking efficiency based on the hybrid picking strategy are greater than those values based on the sequential picking strategy. Furthermore, the fluctuation of instantaneous picking efficiency based on the sequential picking strategy are shaper than that based on the hybrid picking strategy, which means the real time performance of BAOPS is better under the condition of the hybrid picking strategy.

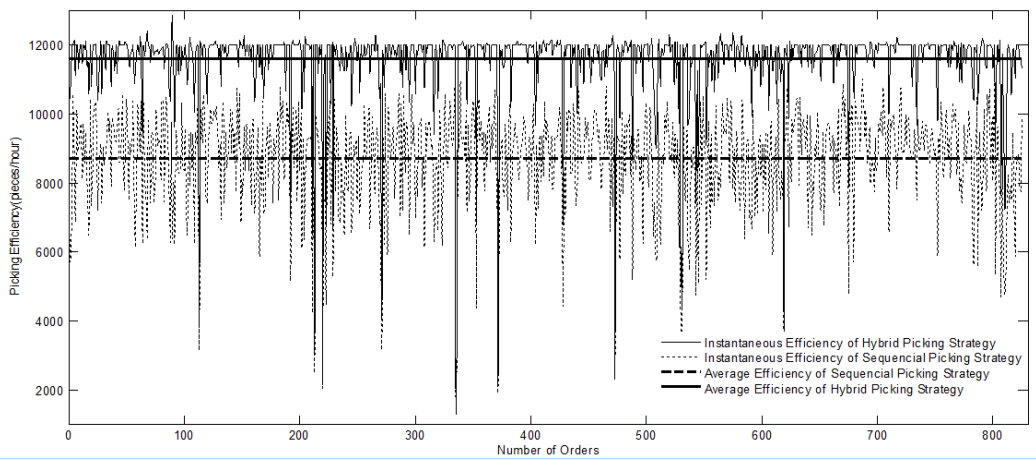

Figure 5. Picking Efficiency of Two Picking Strategies

\section{Conclusion}

In this article, we propose the hybrid picking strategy for BAOPS. This new picking strategy leads to great decrease of waiting time that happens regularly under the condition 
of SPS. Based on results of experimental simulation, the hybrid picking strategy applied to BAOPS results in great increase on picking efficiency and sharp drop on fluctuation of instantaneous picking efficiency. Furthermore, it is beneficial to workload balance and performance stability for BAOPS.

This article has shown the detailed influence on system performance of BAOPS in the view of hybrid picking strategy. It is fascinating to dig out more details about system performance of BAOPS in the view of system parameters. This should be the future research highlights.

\section{Acknowledgements}

This paper is supported by the National Natural Science Foundation of China (Grant No: 61403234), Shandong Province Science and Technology Development Plan (Grant No: 2014GGX106009) and supported by China Postdoctoral Science Foundation (Grant No. 2013M541930).

\section{References}

[1] A. C. Caputo and P. M. Pelagagge, "Management criteria of automated order picking systems in high-rotation high-volume distribution centers", Industrial Management Data Systems, vol.106, no. 9, (2006), pp.1359-1384.

[2] M. E. Johnson and R. D. Meller, "Performance Analysis of Split-Case Sorting Systems", Manufacturing \& Service Operations Management, vol.4, no. 4, (2002), pp. 258-274.

[3] WU Yaohua and ZHANG Yigong, "Order-picking optimization for automated picking system with parallel dispensers", Chinese Journal of Mechanical Engineering, vol. 21, no.6, (2008), pp. 25-29

[4] ZHAO Y., SHI Y. and H. R. Karimi, "Entry-Item-Quantity-ABC Analysis-Based Multitype Cigarette Fast Sorting System", Mathematical Problems in Engineering, vol. 2012, no. 2012, (2012), pp. 1-9

[5] J. A. Pazour and R. D. Meller, "An analytical model for A-frame system design", IIE Transaction, vol. 43, no. 10, (2011), pp. 739-752.

[6] WU Yaohua, ZHANG Yigong and WU Yingying, "Compressible Virtual Window Algorithm in Picking Process Control of Automated Sorting System". Chinese Journal of Mechanical Engineering, vol. 21, no. 3, (2008), pp. 41-45

[7] WU Yingying, WU Yaohua, SHEN Changpeng, "Compressible dynamic virtual window algorithm based on sequential picking strategy", Journal of Shandong University ( Engineering Science), vol. 42, no. 1, (2012), pp. 66-71

[8] LIU Debao, "The research and design of tobacco composting sorting system", Jinan: Shandong University, (2006).

[9] Xiao Jiwei, WU Yaohua, LOU Shanzuo and SUN Guohua, "Optimal Dispensers' Combination for Complex Cigarette Sorting System", Systems Engineering-Theory \& Practice, vol. 30, no. 2, (2010), pp. 251-256.

[10] LIU Peng, WU Yaohua, ZHOU Chen and XU Na, "Fluid-based Slotting Optimization for Automated Order Picking System with Multiple Dispenser Types", Chinese Journal of Mechanical Engineering, vol. 24, no. 4, (2011), pp 529-538

[11] ZHANG Yigong, WU Yaohua and GENG Yaohua, "Item assignment optimization of automated picking system based on synchronized zoning strategy", Computer Engineering and Applications, vol. 47,no. 3, (2011), pp. 240-248

[12] R. D. Meller and J. A. Pazour, "A heuristic for SKU assignment and allocation in an A-Frame system", Proceedings of the 2008 Industrial Engineering Research Conference. (2008), pp. 770-775 
[13] WU Yingying and WU Yaohua, "Order splitting for automated picking system based on parallel Picking Strategy”, Computer Integrated Manufacturing Systems ,vol. 18, no. 10, (2012), pp. 2264-2272

\section{Authors}

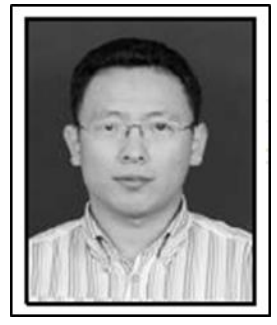

Debao Liu, he is currently a $\mathrm{PhD}$ candidate in School of Control Science and Engineering at Shandong University. He received his B.S., M.S. degree from Shandong University in 2001, 2003, respectively. His research interests include theories of fast sorting system, storage strategy in distribution center.

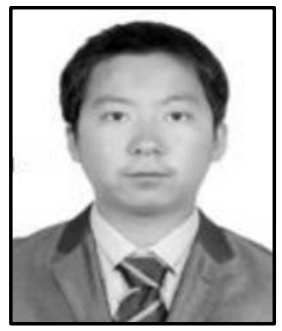

Shandong Mou, he is currently a postgraduate student in School of Control Science and Engineering at Shandong University. He received his B.S. degree majoring in Automation from Shandong University in 2011. His research interests focus on automated order picking system and new storage/retrieval system in logistics distribution center.

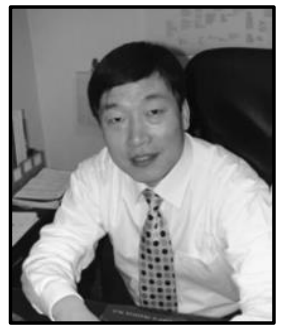

Yaohua $\mathrm{Wu}$, he is Professor of Logistics Engineering in The Logistics Institute at Shandong University. He received the $\mathrm{PhD}$ degree from Tsinghua University in 1996. His research interests are in areas of facilities planning.

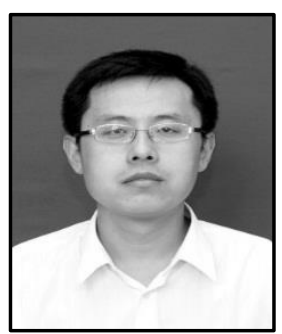

Guofeng Shan, he is an Intermediate Engineer in YANTAI LongYuan Power Technology Co.,LTD. He received his B.S. degree in Shandong University. His research interests are facility design and optimization. 
International Journal of Control and Automation

Vol.8, No.8 (2015) 